\title{
Eotaxin-1 promotes prostate cancer cell invasion via activation of the CCR3-ERK pathway and upregulation of MMP-3 expression
}

\author{
FENG ZHU ${ }^{1}$, PEI LIU ${ }^{1}, \mathrm{JUN}^{2} \mathrm{~L}^{2}$ and YAN $\mathrm{ZHANG}^{3}$ \\ ${ }^{1}$ Department of Urology, The First Affiliated Hospital of Xinxiang Medical University, Weihui 453100; \\ ${ }^{2}$ Department of Urology, The Third Affiliated Hospital of Xinxiang Medical University, Xinxiang 453003; \\ ${ }^{3}$ Department of Physiology, Xinxiang Medical College, Xinxiang, Henan 453003, P.R. China
}

Received November 30, 2013; Accepted January 23, 2014

DOI: $10.3892 /$ or.2014.3060

\begin{abstract}
Chemokines have been reported to play crucial roles in tumor progression. Eotaxin-1 (CCL11), a member of the $\mathrm{CC}$ chemokine family, is elevated in many types of human cancer. Here, to reveal the molecular mechanisms of eotaxin-1 in prostate cancer cell invasion, the expression of eotaxin-1 receptors [CC chemokine receptor (CCR)2, CCR3 and CCR5] were silenced by small interfering RNA (siRNA). The ERK pathway was inhibited by the specific MEK inhibitor U0126. The role of eotaxin-1 and the CCR3-ERK pathway in prostate cancer cell invasion was assessed by invasion and migration assays. MMP-3 expression was detected by real-time PCR and ELISA assay. The results demonstrated that eotaxin-1 promoted the invasion and migration of DU-145 cells, and increased ERK1/2 activation and MMP-3 expression. Knockdown of CCR3 inhibited the invasion and migration of prostate cancer cells, and attenuated the eotaxin-1-induced ERK1/2 activation and MMP-3 expression. Furthermore, inactivation of the ERK pathway suppressed the eotaxin-1-promoted invasion and migration, and decreased MMP-3 expression in the prostate cancer cells. Together, the present study suggests that eotaxin-1 increases MMP-3 expression via the CCR3-ERK pathway, thereby promoting prostate cancer cell invasion and migration. Thus, therapies that block eotaxin-1 and CCR3 may be effective interventions for prostate cancer.
\end{abstract}

\section{Introduction}

Prostate cancer is one of the most common malignant diseases among men, with a high rate of mortality (1). Tumor invasion and metastasis account for the poor survival of prostate cancer patients. During tumor progression, multiple regulatory molecules are released into the tumor microenvironment

Correspondence to: Professor Yan Zhang, Department of Physiology, Xinxiang Medical College, East Hualan Street, Xinxiang, Henan 453003, P.R. China

E-mail: zhangyanvy@126.com

Key words: eotaxin-1, invasion, CCR3, ERK1/2, MMP-3, prostate cancer and play crucial roles in the regulation of tumor invasion and metastasis $(2,3)$. Thus, investigation of these molecules may provide useful clues for the underlying mechanisms of prostate cancer invasion and metastasis.

As a member of the CC chemokine family, eotaxin-1 (CCL11) was initially regarded as an eosinophil chemoattractant, and is involved in the recruitment of inflammatory cells such as eosinophils and neutrophils (4). Overexpression of eotaxin-1 has been found in various inflammatory diseases such as allergic asthma and atopic dermatitis $(5,6)$. Further studies have shown that eotaxin-1 is elevated in many human cancers, and correlates with tumor progression (7). Eotaxin-1 acts mostly via the CC chemokine receptor-3 (CCR3) (8). CCL11-CCR3 interactions have been proven to promote cell survival and growth of anaplastic large cell lymphoma cells (9). In addition to CCR3, a recent study found that CCR2 and CCR5 are also activated by eotaxin-1 (10). Serum eotaxin-1 has been confirmed as a diagnostic marker for prostate cancer (11). Yet, the role of eotaxin-1 in prostate cancer remains elusive. In the present study, we found that eotaxin-1 promotes prostate cancer cell invasion and migration. We also determined the involvement of CC chemokine receptor(s), the ERK pathway and MMP-3 expression in eotaxin-1-mediated prostate cancer cell invasion.

\section{Materials and methods}

Chemicals and antibodies. Eotaxin-1 was purchased from R\&D Systems (Minneapolis, MN, USA). U0126, a MEK inhibitor, was purchased from Sigma (St. Louis, MO, USA). The antibodies against total-ERK1/2 and $\beta$-actin were obtained from Santa Cruz Biotechnology, Inc. (Santa Cruz, CA, USA). The antibody against phosho-ERK1/2 was obtained from Cell Signaling Technology, Inc. (Danvers, MA, USA).

Cell culture. The DU-145 human prostate carcinoma cell line was purchased from the Cell Resource Center of the Chinese Academy of Medical Sciences (Beijing, China). Cells were cultured in RPMI-1640 medium containing 10\% fetal bovine serum (FBS) and incubated in a $\mathrm{CO}_{2}$ incubator at $37^{\circ} \mathrm{C}$.

Real-time PCR. Total mRNA of DU-145 cells was extracted by TRIzol reagent (Invitrogen, Carlsbad, CA, USA). Then 
Table I. Real-time PCR primers.

\begin{tabular}{lllr}
\hline Gene & Forward primer sequence (5'-3') & \multicolumn{1}{c}{ Reverse primer sequence (5'-3') } & Length (bp) \\
\hline MMP-3 & CTGGACTCCGACACTCTGGA & CAGGAAAGGTTCTGAAGTGACC & 79 \\
CCR2 & CCACATCTCGTTCTCGGTTTATC & CAGGGAGCACCGTAATCATAATC & 88 \\
CCR3 & GTCATCATGGCGGTGTTTTTC & CAGTGGGAGTAGGCGATCAC & 155 \\
CCR5 & GTTGGACCAAGCTATGCAGGT & GCAGAAGCGTTTGGCAATGT & 142 \\
$\beta$-actin & GGATGCAGAAGGAGATCACTG & CGATCCACACGGAGTACTTG & 90 \\
\hline
\end{tabular}

cDNA was obtained using the Quantscript cDNA kit (Tiangen, Beijing, China), following the manufacturer's instructions. The cDNA was subjected to real-time PCR with the primers as listed in Table I. The real-time PCR protocol was $10 \mathrm{~min}$ at $95^{\circ} \mathrm{C}$, and 40 cycles of $15 \mathrm{sec}$ at $95^{\circ} \mathrm{C}$ and $1 \mathrm{~min}$ at $60^{\circ} \mathrm{C}$. All expression levels of the examined genes were normalized to the expression of $\beta$-actin and analyzed by the $2^{-\Delta \Delta C t}$ method.

Enzyme-linked immunosorbent assay (ELISA). After treatment with or without eotaxin- 1 for $12 \mathrm{~h}$, the cell supernatant was collected and centrifuged at $12,000 \mathrm{rpm}$ for $15 \mathrm{~min}$. Then the supernatant was subjected to an ELISA kit for human MMP-3 (Boster, Wuhan, China), according to the manufacturer's instructions. Cells were lysed with RIPA buffer, and total protein of the cells was determined by bicinchoninic acid (BCA) assay. Then the concentration of MMP-3 was normalized to the total protein of cells.

Invasion assay and migration assay. Transwell cell culture chambers (Costar, San Diego, CA, USA) were used in the invasion and migration assays. For the invasion assay, the top chambers were coated with Matrigel (Sigma) before use. Next, $1 \times 10^{5}$ cells in $100 \mu 1$ RPMI-1640 were added to the top chambers, while $600 \mu \mathrm{l}$ RPMI-1640 with 20\% FBS was added to the low chambers. After incubated at $37^{\circ} \mathrm{C}$ for $12 \mathrm{~h}$, the invaded cells were fixed with methanol and stained with crystal violet. The number of cells in 7 random visual fields was observed and counted under a microscope at $\times 200$ magnification. Data are presented as a percentage compared to the control group for which the number of invaded cells was defined as $100 \%$. For the migration assay, all procedures were the same as described above except the top chambers were not coated with Matrigel.

Western blotting. Cells were lysed in RIPA buffer with protease inhibitor and phosphatase inhibitor, and the concentration of protein was determined by BCA assay. Equal amounts of protein were separated on SDS-PAGE gel, and then transferred onto PVDF membranes (Bio-Rad, Hercules, CA, USA). The membranes were blocked with 5\% BSA for $1 \mathrm{~h}$, and then incubated with primary antibodies at $4^{\circ} \mathrm{C}$ overnight. After being washed with TBST for 3 times, the membranes were incubated with secondary antibodies for $1 \mathrm{~h}$ at room temperature. Then the bands were visualized and exposed to film by chemiluminescence (Applygen Technologies Inc., Beijing, China).

Small interfering RNAs (siRNAs) and transfection. The CCR2 siRNA, CCR3 siRNA and CCR5 siRNA were obtained from
Santa Cruz Biotechnology, Inc. A scramble siRNA that was also obtained from Santa Cruz Biotechnology was used as the control siRNA. Cells were transfected with siRNAs using Lipofectamine 2000 (Invitrogen). After transfection with siRNAs for $48 \mathrm{~h}$, the knockdown efficiency was tested by real-time PCR, and cells were used in the subsequence experiments.

Statistical analysis. All experiments were repeated three to four times. The means \pm SD was determined and used to represent the results. Statistical significance was set at $p<0.05$ and assessed by Student's t-test (comparison of two means) or non-parametric ANOVA (comparison of multiple means).

\section{Results}

Eotaxin-1 increases the expression of MMP-3 in DU-145 cells. Studies have found that eotaxin-1 can promote MMP-3 expression in inflammatory diseases such as arthritis (12). To determine whether eotaxin- 1 affects the expression of MMP-3 in cancer cells, we first stimulated DU-145 cells with eoxtaxin- 1 for $12 \mathrm{~h}$, and then detected the expression of MMP- 3 by real-time PCR and ELISA assay. The results showed that eotaxin-1 treatment increased the expression of MMP-3 in a dose-dependent manner, and a peak upregulation was noted at $100 \mathrm{ng} / \mathrm{ml}$ (Fig. 1A and B). Thus, eotaxin-1 at the concentration of $100 \mathrm{ng} / \mathrm{ml}$ was used in the subsequent experiments.

Eotaxin-1 promotes the invasion and migration of prostate cancer cells. MMP-3 acts as a pivotal player in the invasion of prostate cancer (13). Therefore, we investigated the effect of eotaxin- 1 on the invasion and migration of prostate cancer cells. Using invasion and migration assays, we found that cells incubated with eotaxin-1 exhibited significantly higher invasion and migration abilities when compared with the control cells, indicating that eotaxin- 1 enhances the invasion and migration of prostate cancer cells (Fig. 2A and B).

Eotaxin-1 induces the activation of ERK1/2 in prostate cancer cells. The ERK pathway is essential to the invasion and metastasis of prostate cancer (14). Here, we detected the phosphorylation of ERK1/2 following eotaxin-1 treatment by western blotting. As shown in Fig. 3, eotaxin-1 stimulated the activation of ERK1/2 in a time-dependent manner, with peak activation at $15 \mathrm{~min}$, suggesting that eotaxin-1 induces the activation of ERK1/2 in prostate cancer cells. 
A

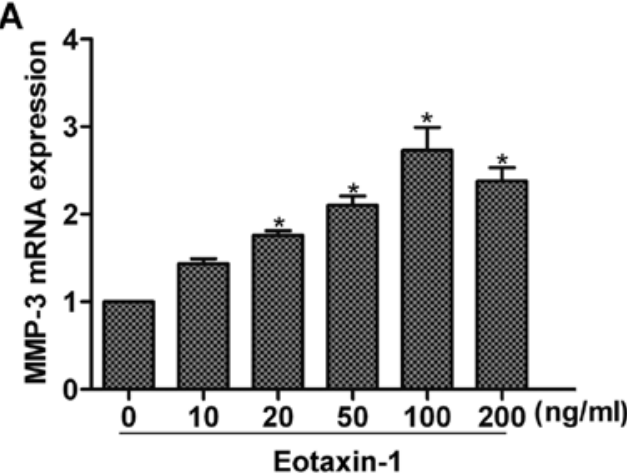

B

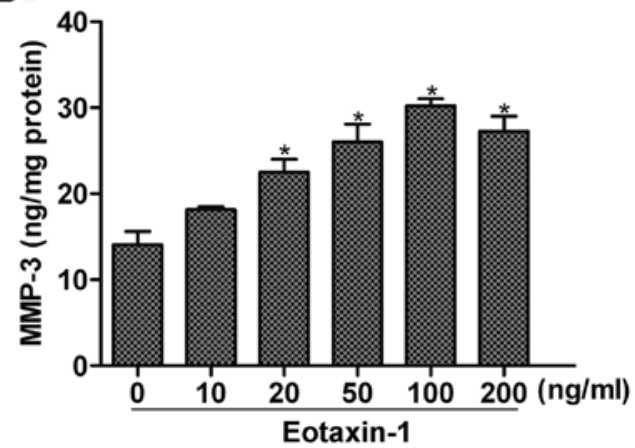

Figure 1. Eotaxin-1 induces MMP-3 expression in DU-145 cells in a dose-dependent manner. DU-145 cells were incubated with eotaxin-1 (10-200 ng/ml) for $12 \mathrm{~h}$, and the expression of MMP-3 was examined by (A) real-time PCR and (B) ELISA assay. Data are presented as means \pm SD. "p $<0.05$.

A

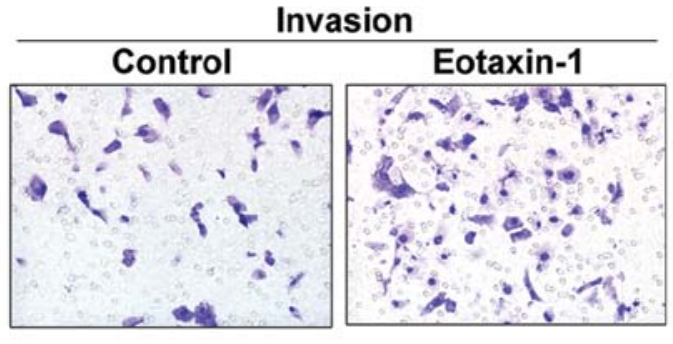

B

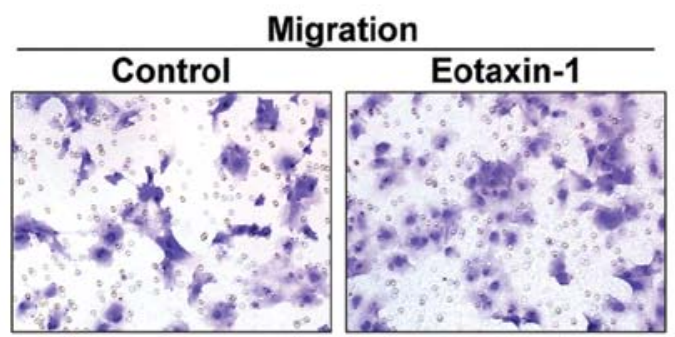

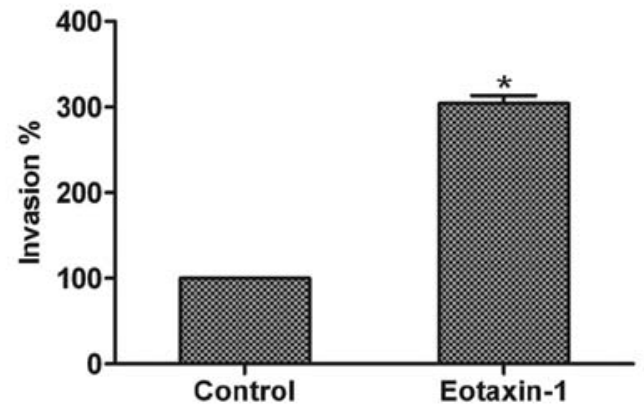

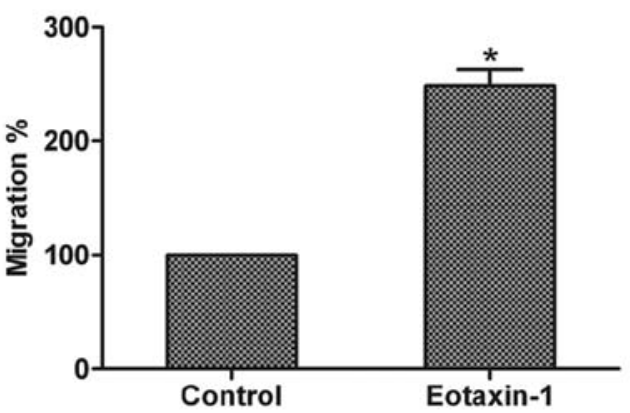

Figure 2. Eotaxin-1 promotes the invasion and migration of DU-145 cells. (A) Effect of eotaxin-1 treatment (100 ng/ml) on the invasion of DU-145 cells. (B) Effect of eotaxin-1 treatment $(100 \mathrm{ng} / \mathrm{ml})$ on the migration of DU-145 cells. Data are presented as means \pm SD. ${ }^{*} \mathrm{p}<0.05$.
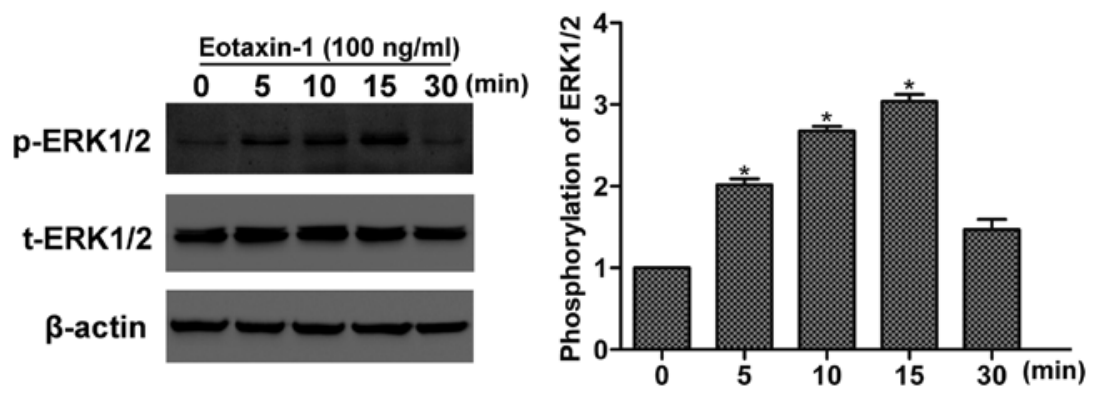

Figure 3. Eotaxin-1 induces the activation of ERK1/2 in a time-dependent manner. Cells were treated with eotaxin-1 (100 ng/ml), and the phosphorylation of ERK1/2 was detected throughout a 30 -min period. Data are presented as means \pm SD. " $\mathrm{p}<0.05$.

CCR 3 is required for the eotaxin-1-promoted invasion and migration. CCR2, CCR3 and CCR5 are the receptors that respond to eotaxin-1 treatment (10). Thus, we attempted to ascertain whether these receptors are involved in the eotaxin1-promoted invasion and migration by siRNA technology. The knockdown efficiency of CCR2, CCR3 and CCR5 siRNAs 

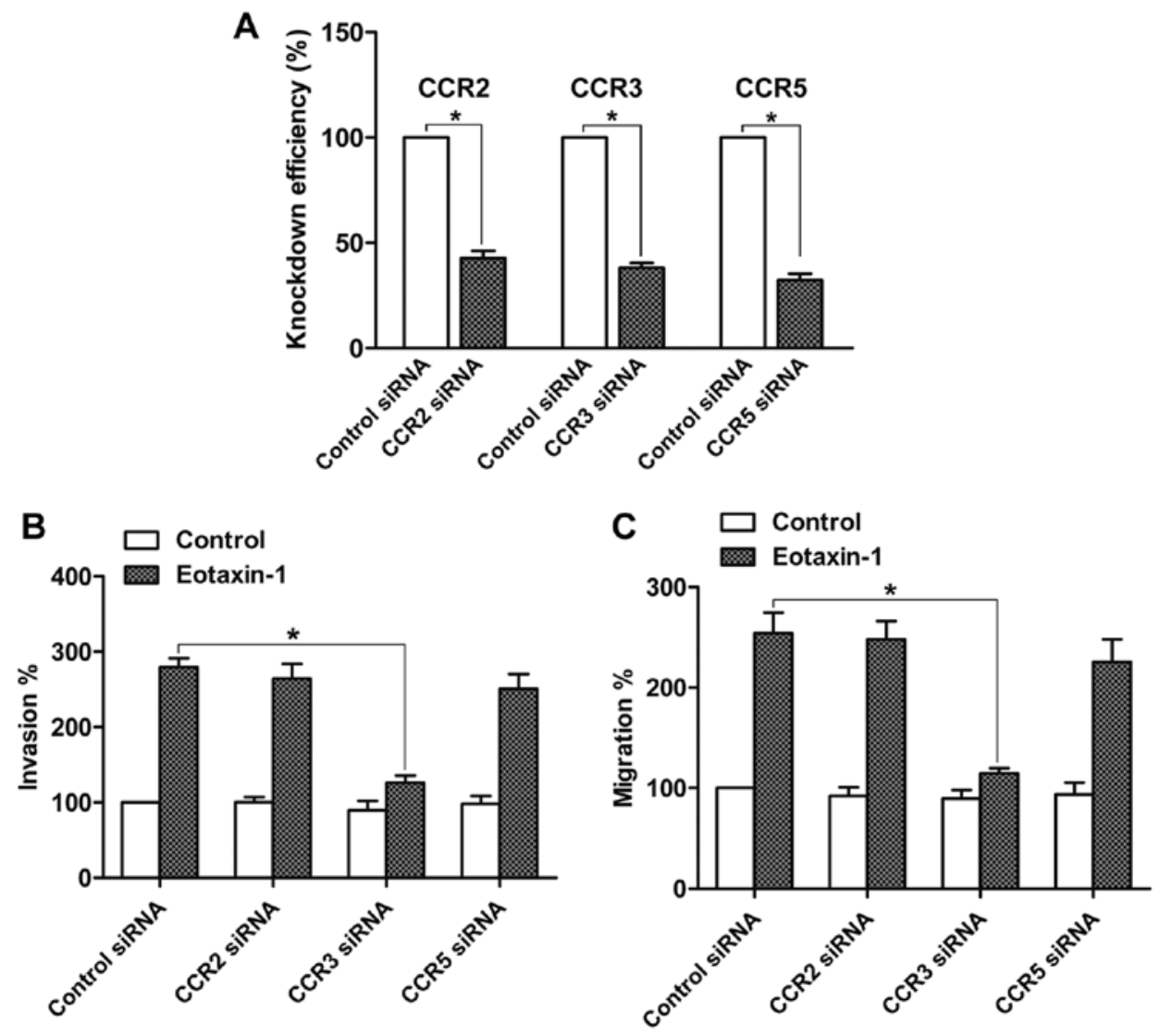

Figure 4. Knockdown of CCR3 suppresses the eotaxin-1-promoted invasion and migration of DU-145 cells. DU-145 cells were transfected with CCR2 siRNA, CCR3 siRNA, CCR5 siRNA and control siRNA, respectively. (A) The knockdown efficiency was tested by real-time PCR. Cells were treated with or without eotaxin-1 and subjected to (B) invasion and (C) migration assays. Data are presented as means \pm SD. . $\mathrm{p}<0.05$.

was examined by real-time PCR, and each siRNA achieved a prominent knockdown effect when compared with the control siRNA (Fig. 4A). Then invasion and migration assays were carried out. The results demonstrated that knockdown of CCR3 suppressed the eotaxin-1-mediated invasion and migration of DU-145 cells. However, neither CCR2 nor CCR5 knockdown affected the eotaxin-1-mediated invasion and migration of DU-145 cells (Fig. 4B and C). These data suggest that eotaxin-1 promotes prostate cancer cell invasion and migration via CCR3.

Involvement of CCR3 in eotaxin-1-regulated ERK1/2 activation and MMP-3 expression. To explore the involvement of CCR3 in eotaxin-1-induced ERK1/2 activation, we silenced the expression of CCR3 by siRNA and incubated DU-145 cells with eotaxin-1 for $15 \mathrm{~min}$. Then we detected the phosphorylation of ERK1/2 by western blotting. The results showed that CCR3 knockdown inhibited the eotaxin-1-induced activation of ERK1/2 (Fig. 5A). To determine the function of CCR3 in eotaxin-1-promoted MMP-3 expression, the expression of CCR3 was knocked down and the cells were incubated with eotaxin-1 for $12 \mathrm{~h}$. Next, we examined the expression of MMP-3 by real-time PCR and ELISA assay. We found that the knockdown of CCR3 decreased the eotaxin-1-regulated MMP-3 expression at both the mRNA and protein levels (Fig. 5B and C). These results indicate that CCR3 is involved in the eotaxin-1-regulated ERK1/2 activation and MMP-3 expression.
The ERK pathway is required for the eotaxin-1-promoted invasion and MMP-3 expression. To explore the role of the ERK pathway in eotaxin-1-promoted invasion, we pretreated DU-145 cells with the MEK inhibitor U0126 $(10 \mu \mathrm{M})$ for 30 min to inhibit the activation of the ERK pathway. Then we subjected the cells to invasion and migration assays with or without eotaxin-1 treatment. The results showed that U0126 was able to inhibit the eotaxin-1-promoted prostate cancer cell invasion and migration (Fig. 6A and B), confirming that the ERK pathway participates in the eotaxin-1-promoted invasion and migration of prostate cancer cells. Furthermore, we pretreated DU-145 cells with U0126 $(10 \mu \mathrm{M})$ or DMSO (control) for $30 \mathrm{~min}$, and then incubated the cells with eotaxin-1 for $12 \mathrm{~h}$. Using real-time PCR and ELISA assay, we found that U0126 suppressed the eotaxin-1-mediated expression of MMP-3, suggesting that the eotaxin-1-mediated increase in MMP-3 expression involved the ERK pathway (Fig. 6C and D).

\section{Discussion}

As a member of the $\mathrm{CC}$ chemokine family, eotaxin- 1 is able to promote cell migration in vitro, and to induce angiogenesis in vivo (15-17). Recently published studies have found that eotaxin-1 is upregulated in many tumor types such as prostate (11) and breast cancer (18), indicating the involvement of eotaxin-1 in tumor progression. Here, our results demonstrated that eotaxin-1 may promote prostate cancer cell invasion via the CCR3-ERK pathway and MMP-3 expression. These 
A

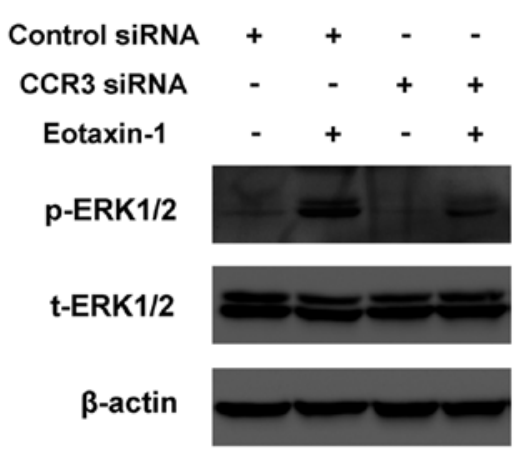

B

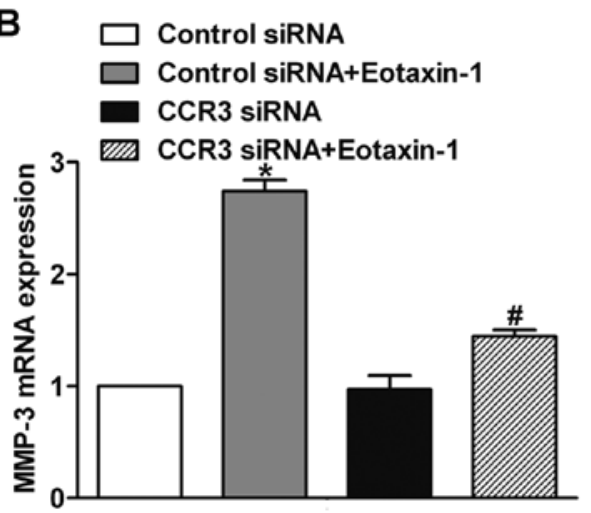

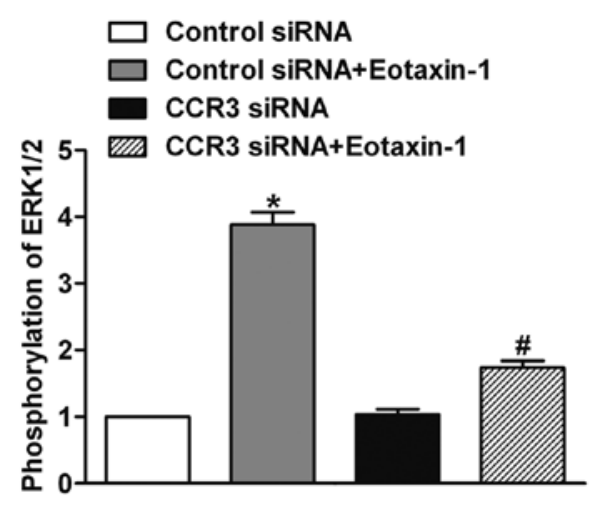

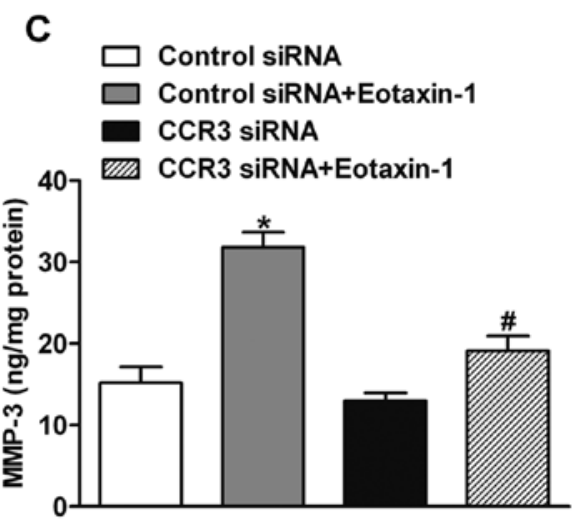

Figure 5. Knockdown of CCR3 inhibits the eotaxin-1-induced ERK1/2 activation and MMP-3 expression in DU-145 cells. (A) After transfection with siRNAs for $48 \mathrm{~h}$, DU-145 cells were treated with eotaxin- 1 for $15 \mathrm{~min}$, and the phosphorylation of ERK1/2 was detected by western blotting. After knockdown of CCR3, DU-145 cells were incubated with eotaxin-1 for $12 \mathrm{~h}$. (B) Real-time PCR and (C) ELISA assay were performed to examine the expression of MMP-3. Data are presented as means $\pm \mathrm{SD}$. ${ }^{\mathrm{p}}<0.05$ vs. control siRNA, ${ }^{\#} \mathrm{p}<0.05$ vs. control siRNA+eotaxin-1.
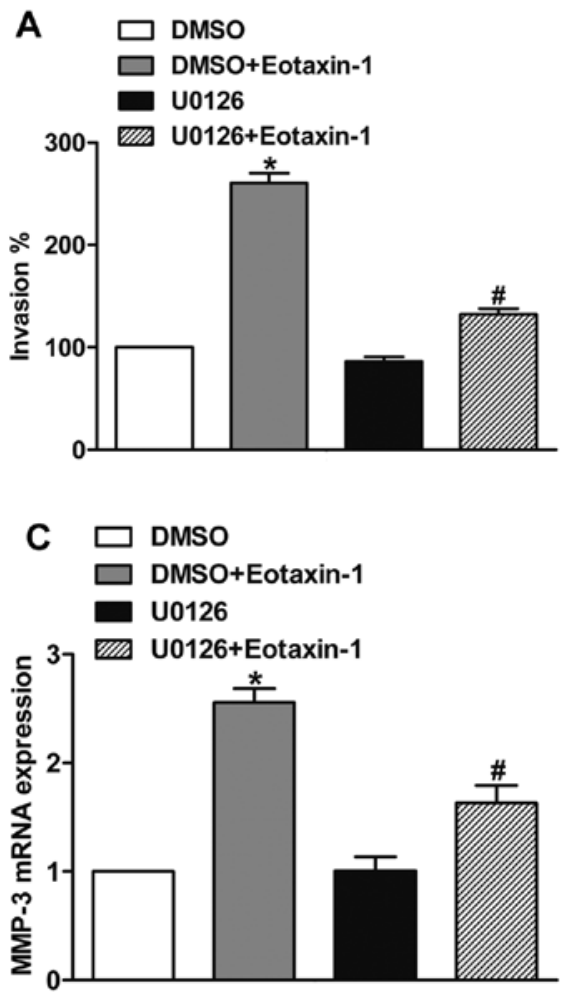

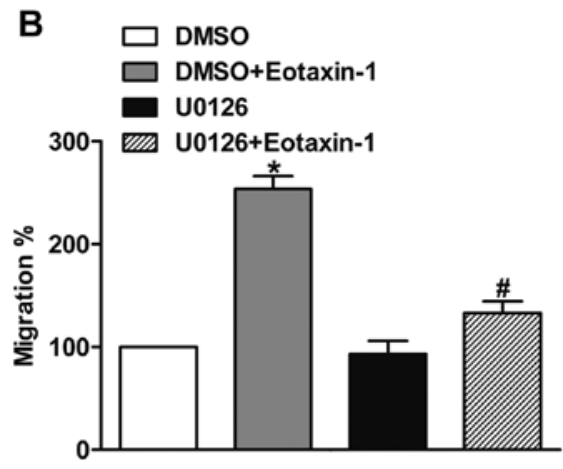

D

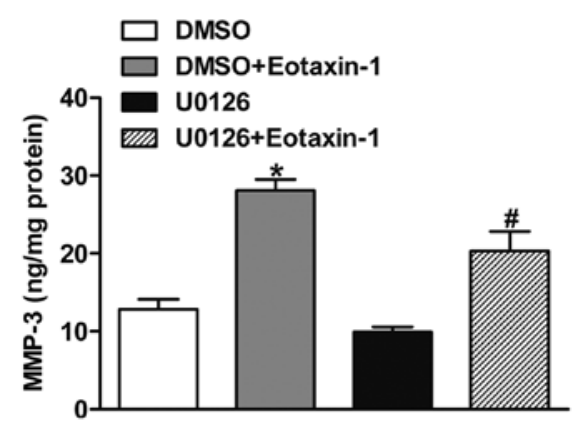

Figure 6. Inhibition of the ERK pathway suppresses the eotaxin-1-mediated invasion and MMP-3 expression of prostate cancer cells. DU-145 cells were pretreated with DMSO or U0126 for 30 min, and incubated with or without eotaxin-1. (A) Effect of U0126 on the eotaxin-1-promoted invasion of DU-145 cells. (B) Effect of U0126 on the eotaxin-1-promoted migration of DU-145 cells. (C) Real-time PCR and (D) ELISA assay were carried out to detect the expression of MMP-3. Data are presented as means \pm SD. ${ }^{*} \mathrm{p}<0.05$ vs. DMSO, ${ }^{*} \mathrm{p}<0.05$ vs. DMSO+eotaxin-1. 
findings strongly support the notion that eotaxin-1 is a crucial player in the regulation of prostate cancer cell invasion.

As the major receptor for eotaxin-1, CCR3 was found to be upregulated in human renal cell carcinoma and glioblastoma $(19,20)$. Activation of CCR3 by eotaxin-1 enhanced the migration of choroidal endothelial cells and smooth muscle cells $(15,21)$. In the present study, we found that eotaxin-1 promoted the invasion and migration of prostate cancer cells via CCR3. CCR3 has been proven to activate the ERK pathway in smooth muscle cells and large cell lymphoma cells $(9,22)$. Consistent with that result, we found that CCR3 was involved in the eotaxin-1-induced activation of ERK1/2 in DU-145 cells. It is well established that the ERK pathway plays major roles in a series of tumor progression processes such as proliferation, invasion and metastasis (23). Here, our results demonstrated that the ERK pathway is required for the eotaxin-1-promoted invasion and migration of prostate cancer cells.

MMP-3 is a member of the matrix metalloproteinases (MMPs), and is essential to the invasion and metastasis of human cancers (24). Studies have found that eotaxin-1 treatment upregulates the expression of MMP-3 in chondrocytes (25). However, whether eotaxin-1 treatment affects MMP-3 expression of cancer cells is still unclear. Here, we found that eotaxin-1 increased the expression of MMP-3 in DU-145 cells. Since degradation of the extracellular matrix by MMPs is a prerequisite for tumor invasion and metastasis (26), our finding indicates that MMP-3 may participate in the eotaxin-1-promoted prostate cancer cell invasion. Studies have shown that upregulation of MMP-3 by eotaxin-1 treatment in human chondrocytes is dependent on ERK1/2 activation (12). In the present study, our results showed that eotaxin-1 promoted the expression of MMP-3 via the CCR3-ERK pathway.

In conclusion, the present study demonstrated that eotaxin-1 promotes the invasion and migration of prostate cancer cells. Activation of the CCR3-ERK pathway and upregulation of MMP-3 expression were involved in these processes. In vivo studies are required to further determine the role of eotaxin-1 and CCR3 in prostate cancer.

\section{References}

1. Siegel R, Naishadham D and Jemal A: Cancer statistics, 2013. CA Cancer J Clin 63: 11-30, 2013.

2. Chung LWK, Baseman A, Assikis V and Zhau HE: Molecular insights into prostate cancer progression: the missing link of tumor microenvironment. J Urol 173: 10-20, 2005.

3. Harlozinska A: Progress in molecular mechanisms of tumor metastasis and angiogenesis. Anticancer Res 25: 3327-3333, 2005.

4. Menzies-Gow A, Ying S, Sabroe I, et al: Eotaxin (CCL11) and eotaxin-2 (CCL24) induce recruitment of eosinophils, basophils, neutrophils, and macrophages as well as features of early- and late-phase allergic reactions following cutaneous injection in human atopic and nonatopic volunteers. J Immunol 169: 2712-2718, 2002.

5. Campbell EM, Kunkel SL, Strieter RM and Lukacs NW: Temporal role of chemokines in a murine model of cockroach allergen-induced airway hyperreactivity and eosinophilia. J Immunol 161: 7047-7053, 1998.
6. Yawalkar N, Uguccioni M, Schärer J, et al: Enhanced expression of eotaxin and CCR3 in atopic dermatitis. J Invest Dermatol 113: 43-48, 1999.

7. Nolen BM and Lokshin AE: Targeting CCL11 in the treatment of ovarian cancer. Expert Opin Ther Targets 14: 157-167, 2010.

8. Uguccioni M, Mackay CR, Ochensberger B, et al: High expression of the chemokine receptor CCR3 in human blood basophils. Role in activation by eotaxin, MCP-4, and other chemokines. J Clin Invest 100: 1137-1143, 1997

9. Miyagaki T, Sugaya M, Murakami T, et al: CCL11-CCR3 interactions promote survival of anaplastic large cell lymphoma cells via ERK1/2 activation. Cancer Res 71: 2056-2065, 2011.

10. Levina V, Nolen BM, Marrangoni AM, et al: Role of eotaxin-1 signaling in ovarian cancer. Clin Cancer Res 15: 2647-2656, 2009.

11. Agarwal M, He C, Siddiqui J, Wei JT and Macoska JA: CCL11 (eotaxin-1): a new diagnostic serum marker for prostate cancer. Prostate 73: 573-581, 2013.

12. Chao PZ, Hsieh MS, Cheng CW,Lin YF and Chen $\mathrm{CH}$ : Regulation of MMP-3 expression and secretion by the chemokine eotaxin-1 in human chondrocytes. J Biomed Sci 18: 86, 2011.

13. Lein M, Nowak L, Jung K, Koenig F, Schnorr D and Loening SA: Metalloproteinases (MMP-1, MMP-3) and their inhibitors (TIMP) in blood plasma of patients with prostate carcinoma. Urologe A 37: 377-381, 1998 (In German).

14. Zhu G, Zhou J, Song W, et al: Role of GLI-1 in epidermal growth factor-induced invasiveness of ARCaPE prostate cancer cells. Oncol Rep 30: 904-910, 2013.

15. Kodali RB, Kim WJ, Galaria, II, et al: CCL11 (Eotaxin) induces CCR3-dependent smooth muscle cell migration. Arterioscler Thromb Vasc Biol 24: 1211-1216, 2004.

16. Salcedo R, Young HA, Ponce ML, et al: Eotaxin (CCL11) induces in vivo angiogenic responses by human $\mathrm{CCR}^{+}{ }^{+}$endothelial cells. J Immunol 166: 7571-7578, 2001.

17. Muessel MJ, Scott KS, Friedl P, Bradding P and Wardlaw AJ: CCL11 and GM-CSF differentially use the Rho GTPase pathway to regulate motility of human eosinophils in a three-dimensional microenvironment. J Immunol 180: 8354-8360, 2008.

18. Georgiou GK, Igglezou M, Sainis I, et al: Impact of breast cancer surgery on angiogenesis circulating biomarkers: a prospective longitudinal study. World J Surg Oncol 11: 213, 2013.

19. Jöhrer K, K, Zelle-Rieser C, Perathoner A, et al: Up-regulation of functional chemokine receptor CCR3 in human renal cell carcinoma. Clin Cancer Res 11: 2459-2465, 2005.

20. Kouno J, Nagai H, Nagahata T, et al: Up-regulation of CC chemokine, CCL3L1, and receptors, CCR3, CCR5 in human glioblastoma that promotes cell growth. J Neurooncol 70: 301-307, 2004.

21. Wang H, Wittchen ES, Jiang Y, Ambati B, Grossniklaus HE and Hartnett ME: Upregulation of CCR3 by age-related stresses promotes choroidal endothelial cell migration via VEGF-dependent and -independent signaling. Invest Ophthalmol Vis Sci 52: 8271-8277, 2011.

22. Markwick LJ, Clements D, Roberts ME, Ceresa CC, Knox AJ and Johnson SR: CCR3 induced-p42/44 MAPK activation protects against staurosporine induced-DNA fragmentation but not apoptosis in airway smooth muscle cells. Clin Exp Allergy 42: 1040-1050, 2012

23. Reddy KB, Nabha SM and Atanaskova N: Role of MAP kinase in tumor progression and invasion. Cancer Metastasis Rev 22: 395-403, 2003.

24. Chakraborti S, Mandal M, Das S, Mandal A and Chakraborti T: Regulation of matrix metalloproteinases: an overview. Mol Cell Biochem 253: 269-285, 2003.

25. Hsu YH, Hsieh MS, Liang YC, et al: Production of the chemokine eotaxin-1 in osteoarthritis and its role in cartilage degradation. J Cell Biochem 93: 929-939, 2004.

26. Bachmeier BE,Nerlich AG, Lichtinghagen R and Sommerhoff CP: Matrix metalloproteinases (MMPs) in breast cancer cell lines of different tumorigenicity. Anticancer Res 21: 3821-3828, 2001. 